\title{
EPIDEMIOLOGÍA DE LA RETINOPATÍA DIABÉTICA EN PACIENTES TIPO II. CAMBIOS OBSERVADOS EN UNA POBLACIÓN ENTRE LOS AÑOS 1993 Y 2005, TRAS LOS NUEVOS CRITERIOS DIAGNÓSTICOS Y UN MAYOR CONTROL DE LOS PACIENTES
}

\section{DIABETIC RETINOPATHY EPIDEMIOLOGY IN TYPE II DIABETIC PATIENTS. EFFECT OF THE CHANGES IN THE DIAGNOSTIC CRITERIA AND STRICTER CONTROL OF THE DIABETES BETWEEN 1993 AND 2005 ON THE INCIDENCE OF DIABETIC RETINOPATHY}

\section{ROMERO-AROCA P ${ }^{1}$, FERNÁNDEZ-ALART J ${ }^{2}$, BAGET-BERNALDIZ $\mathrm{M}^{3}$, MÉNDEZ-MARÍN I ${ }^{4}$, SALVAT-SERRA $\mathrm{M}^{3}$}

\section{RESUMEN}

Objetivo: Comparar los resultados obtenidos en una muestra de población de 741 pacientes diabéticos tipo II en el año 1993 con una muestra actual de 741 pacientes diabéticos tipo II, determinando la prevalencia de retinopatía diabética, y valorando el impacto sobre la misma de los nuevos criterios diagnósticos y el mayor control de los pacientes diabéticos.

Métodos: Estudio transversal en el que la muestra fue recogida mediante selección al azar de 741 pacientes afectos de diabetes mellitus tipo II del total del archivo de pacientes visitados regularmente a lo largo del período comprendido entre el 1 de enero y el 31 de diciembre del año 2005.

Resultados: Se observa un descenso de prevalencia de retinopatía diabética del $39,41 \%$ en el primer

\begin{abstract}
Objective: The aim of the study was to compare the results with those of a previous study by the same author in 1993 when 741 type II diabetic patients were recruited. We determined the prevalence of diabetic retinopathy and the impact of the new diagnostic criteria and stricter control of diabetes on the results obtained.

Methods: The study sample was obtained by hazard selection of 741 type II diabetic patients, from the total diabetic patients visited in the interval between January 1 and December 1 in 2005 .

Results: We observed a decrease in the prevalence of diabetic retinopathy between the two studies. In the first study the incidence was $39.41 \%$ while in the present study it was $27.55 \%$. The diabetic macular edema prevalence was similar in both stu-
\end{abstract}

\footnotetext{
Recibido: 28/4/07. Aceptado: 26/3/07.

Servicio de Oftalmología. Hospital Universitario Sant Joan de Reus. Departamento de Medicina y Cirugía. Universidad Rovira y Virgili.

1 Doctor en Medicina. Servicio de Oftalmología. Universidad Rovira i Virgili.

2 Doctor en Medicina. Servicio de Epidemiología. Universidad Rovira i Virgili.

3 Licenciado en Medicina.

4 Doctor en Medicina.
}

Correspondencia:

Pedro Romero Aroca

Cl. Ample, 55, 1. ${ }^{\circ}$

43202 Reus (Tarragona)

España

E-mail: promero@grupsagessa.com 
estudio al $27,55 \%$ actual, manteniéndose igual el edema macular $(7,15 \%$ en el primer estudio y $7,90 \%$ en el actual). Se ha observado un descenso en el número de pacientes ciegos, de un 11,20\% a un $4,90 \%$. Asimismo los pacientes tratados previamente con fotocoagulación láser aumentaron en el estudio actual, un $13,49 \%$ frente a un 6,20\%. En el estudio estadístico los factores de riesgo son: el tiempo de evolución de la diabetes, el tratamiento con insulina de la misma, y los niveles elevados de HbA1c.

Conclusiones: El mayor control ejercido sobre los pacientes diabéticos nos permite observar una mejoría en la agudeza visual. Observándose también que aumenta el número de pacientes tratados. Por otro lado al existir un mayor número de pacientes diabéticos conocidos, se produce un efecto de disminución de la prevalencia de la retinopatía.

Palabras clave: Retinopatía diabética, diabetes mellitus tipo II, edema macular diabético, epidemiología de la retinopatía diabética. dies $(7.15 \%$ in the past and $7.90 \%$ in the present study). There was also a decrease in the number of blind patients $(11.20 \%$ in 1993 and $4.90 \%$ in the current study). The number of patients treated with laser photocoagulation increased $(13.49 \%$ in the current study as compared to $6.20 \%$ in the previous study). Statistic analysis revealed the risk factors for retinopathy: diabetes mellitus duration, elevated $\mathrm{HbA} 1 \mathrm{C}$ levels and the need for insulin treatment.

Conclusions: A better control of diabetes mellitus may lead us to observe an increase in visual acuity, and a better control of diabetic retinopathy. The incidence of diabetic retinopathy certainly decreased between the study periods; however the overall incidence of diabetes in the community has increased during the last few years, making firm conclusions difficult (Arch Soc Esp Oftalmol 2007; 82: 209-218).

Key words: Diabetic retinopathy, Type II Diabetes Mellitus, diabetic macular edema, diabetic retinopathy epidemiology.

\section{INTRODUCCIÓN}

Los estudios epidemiológicos sobre retinopatía diabética publicados en nuestro país, han dando cifras de prevalencia de retinopatía diabética que oscilan entre un $20,9 \%$ y un $51,4 \%$ (1-14), la razón de dicha disparidad es que se han hecho en épocas diferentes y algunos son series clínicas y no se han basado en estudios poblacionales. Además se debe tener en cuenta que algunos son anteriores al año 1998, año en que la Organización Mundial de la Salud (OMS) modificó los criterios diagnósticos y de clasificación de la diabetes mellitus.

La OMS publicó las primeras recomendaciones sobre diagnóstico y clasificación de la diabetes mellitus en el año 1980, siendo actualizada en el año 1985 (15). El criterio diagnóstico de diabetes mellitus se establecía en $140 \mathrm{mg} / \mathrm{dL}$ de glucosa como cifra límite en ayunas. A partir de 1998 la OMS estableció como nueva cifra diagnóstica de diabetes la glicemia en ayunas igual o mayor de 126 $\mathrm{mg} / \mathrm{dL}$, asimismo se editaba una nueva clasificación de la diabetes (16).

A la aplicación de estos nuevos criterios diagnósticos, que han modificado sin duda la prevalencia de las complicaciones de la diabetes mellitus, se han añadido en los últimos años la aplicación de los resultados obtenidos sobre el control estricto de la glicemia, ya que diversos estudios multicéntricos como el DCCT (17) y el UKPDSG (18), pusieron de manifiesto que retrasaba la aparición de las complicaciones de la diabetes mellitus como la retinopatía diabética.

En el Servicio de Oftalmología de nuestro Hospital, se puso en marcha desde el año 1990 un plan de seguimiento de los pacientes diabéticos de nuestra área, obteniendo en el año 1993 los primeros datos epidemiológicos de la afectación ocular en forma de retinopatía diabética $(6,8)$.

El objetivo del presente estudio es comparar los resultados obtenidos en una muestra de población de 741 pacientes afectos de diabetes mellitus tipo II en el año 1993 con una muestra actual de 741 pacientes diabéticos tipo II (año 2006), a través de un estudio de prevalencia de retinopatía diabética, valorando el impacto sobre la misma de los nuevos criterios diagnósticos de la diabetes mellitus (criterios de la OMS de 1998), así como si el control más estricto de los pacientes (recomendaciones emanadas del estudio DCCT y UKPDSG) ha llevado a una mejoría en los parámetros oculares de los pacientes. 


\section{SUJETOS, MATERIAL Y MÉTODOS}

\section{Diseño del estudio}

El Hospital Universitario Sant Joan de Reus es el único existente en nuestra área y de él depende una población de 175.410 habitantes (se puede clasificar el área en urbana y semiurbana a la vez), de los cuales se calcula que un $6,4 \%$ de la población con edad superior a 30 años (19), presentan diabetes mellitus tipo II (6.212 pacientes). La exploración del fondo de ojo de dichos pacientes se realiza mediante una visita anual en el Servicio de Oftalmología del Hospital, en la unidad de retina y vítreo del mismo, siendo derivados desde las diversas Áreas Básicas por los médicos de atención primaria, y desde la unidad de endocrinología del Hospital. En dicha Unidad se tienen censados actualmente 3.720 pacientes que se visitan puntualmente en dicho Servicio. Los criterios diagnósticos de diabetes mellitus aplicados en las Áreas Básicas dependientes de nuestro Hospital incluyen las recomendaciones de la OMS del año 1998.

El presente estudio transversal de prevalencia, se realizó empleando la misma metodología que el primer estudio, mediante la selección al azar de 741 pacientes afectos de diabetes mellitus tipo II del total del archivo de pacientes visitados regularmente a lo largo del periodo comprendido entre el 1 de enero y el 31 de diciembre del año 2005.

Los pacientes que fueron recogidos en la primera muestra del año 1993 han sido excluidos del presente estudio.

\section{Potencia del estudio}

La muestra del estudio se ha obtenido mediante la aplicación de la fórmula con corrección para poblaciones finitas, con un riesgo prefijado de un $95 \%$ y un intervalo o precisión de un $3 \%$, siendo necesarios según dicha fórmula la obtención de una muestra de 672 pacientes, se ha completado hasta 741 pacientes para poder comparar mejor la muestra actual con la obtenida el año 1993 que era de 741 pacientes, y a su vez poder estratificar la misma según los factores de riesgo epidemiológico como el sexo, edad y tiempo de evolución de la diabetes mellitus.

\section{Examen de los pacientes}

A todos los enfermos se les realizó una historia clínica completa y exploración oftalmológica básica, determinando la agudeza visual con optotipos E de Snellen, presión intra-ocular mediante tonómetro de aplanación, exploración de los segmentos anterior y posterior. Se consideró ceguera legal si la visión en el mejor ojo era inferior o igual a 0,1 , y baja visión si la agudeza visual estaba comprendida entre 0,2 y 0,4 en la escala de Snellen.

El diagnóstico de retinopatía diabética se realizó mediante retinografías de fondo de ojo en dos campos de $50^{\circ}$ uno centrado en mácula y otro centrado a nivel nasal de la papila mediante angiografo TOPCON TRC NW 7SF (20). Se realizaron angiografías fluoresceínicas a aquellos pacientes que lo precisaron, la interpretación de las mismas fue practicada siempre por el mismo oftalmólogo.

Se registraron y analizaron también los siguientes factores de riesgo: sexo, edad, tiempo de evolución de la diabetes mellitus (agrupada en inferior a 15 años y superior a 15 años), presencia de hipertensión arterial (definiéndose como tal el diagnóstico previo de la misma realizado por su médico de atención, control metabólico mediante la determinación del valor medio de la HbA1c obtenida cada tres meses en el último año anterior al estudio, determinación del nivel de colesterol-LDL, y determinación de microalbumina en orina de 24 horas.

\section{Criterios diagnósticos}

La diabetes mellitus se diagnosticó por los médicos de atención primaria, según la recomendación hecha por la OMS en el año 1998 (16).

El diagnóstico de edema macular diabético se ha realizado mediante la aplicación de los criterios recomendados por el Early Treatment Diabetic Retinopathy Study Research Group (ETDRS) (21).

La retinopatía se clasificó atendiendo a la recomendación de la Academia Americana de Oftalmología del año 2000 (22), si bien dado que se tiene que comparar con el estudio epidemiológico realizado en el año 1993, se ha comparado el tipo de retinopatía diabética que presentan los pacientes según la siguiente forma:

- Las formas de retinopatía diabética leve y moderada se han comparado con la forma intrarretiniana.

- Las retinopatía severa se ha comparado con la retinopatía pre-proliferativa. 
- La retinopatía proliferativa es la misma para los dos tipos de clasificaciones, por tanto no se ha precisado ninguna subclasificación especial.

El edema macular diabético se clasificó en focal y difuso. Los pacientes con fotocoagulación focal realizada previamente se han clasificado como edema macular focal; igualmente los pacientes con panfotocoagulación láser realizada previamente se han clasificado según la forma de retinopatía diabética que llevo a indicar dicho tratamiento láser.

\section{Estudio estadístico}

El estudio estadístico se realizó con el programa estadístico SPSS-Windows 13.0 para Windows (SPSS Inc. Chicago, Illinois) considerándose como significativos aquellos valores igual o inferior a 0.05. El análisis comparativo de medias se ha expresado en forma de media \pm desviación standart mediante la aplicación del estadístico t de Student para los valores cuantitativos y la aplicación del test de Chi-cuadrado para los valores cualitativos, determinándose el riesgo relativo para cada factor de riesgo evaluado con sus intervalos de confianza (IC 95\%). Finalmente se ha realizado un estudio multivariante mediante la aplicación de la regresión logística, en la que se han introducido los distintos factores de riesgo estudiados, determinándose su significación estadística así como el riesgo relativo para cada uno de ellos.

\section{RESULTADOS}

La edad media de los pacientes fue de 65,50DE10,99 años, según el sexo: 410 mujeres
$(55,30 \%)$ y 331 hombres $(44,70 \%)$; el tiempo medio de evolución de la diabetes mellitus fue de 10,42DE8,30años. La presencia de hipertensión arterial se asoció en 507 pacientes $(68,42 \%)$.

En el primer estudio (1993) la edad media de los pacientes fue de 62,03DE10,59 años, según el sexo: 412 mujeres $(55,60 \%)$ y 329 hombres $(44,40 \%)$; el tiempo medio de evolución de la diabetes mellitus fue de 9,76DE8,33 años. La presencia de hipertensión arterial se asoció en 499 (67,34\%).

Las diferencias observadas atendiendo a estos factores (sexo, edad, tiempo de evolución y hipertensión arterial) entre ambos estudios, no fueron significativas estadísticamente tras la aplicación de la t de Student (tabla I).

En la tabla I se puede asimismo observar las diferencias respecto al tratamiento de la diabetes mellitus, entre ambos grupos, en el presente estudio se ha observado una menor presencia de pacientes controlados únicamente con dieta, respecto al estudio del año 1993; a su vez el número de pacientes tratados con antidiabéticos orales es superior en el actual estudio; de todas maneras si se compara el número de pacientes tratados con insulina versus los no-insulino-tratados, las diferencias no son significativas en la aplicación del estadígrafo t-Student $(\mathrm{p}=0,130)$.

\section{Estudio de la agudeza visual}

La agudeza visual (tabla II) de los pacientes al finalizar el estudio fue inferior o igual a 0,1 (ceguera legal secundaria a la retinopatía diabética) en el mejor ojo en 36 pacientes $(4,90 \%)$, los pacientes con baja visión secundaria a la presencia de retino-

Tabla I. Datos demográficos del estudio

\begin{tabular}{lccc}
\hline & Presente estudio & Estudio del año 1993 & Significación* \\
\hline Hombre/ Mujer & $331 / 410$ & $329 / 411$ & 0,213 \\
& $(55,30 \% / 44,70 \%)$ & $(55,60 \% / 44,40 \%)$ & 0,187 \\
Edad en años (media \pm DS) & $65,50 \pm 10,99$ & $62,03 \pm 10,59$ & 0,08 \\
Tiempo de evolución de la diabetes en años (media \pm DS) & $10,42 \pm 8,30$ & $9,76 \pm 8,33$ & 0,387 \\
Presencia de hipertensión arterial & $507(68,42 \%)$ & $499(67,34 \%)$ & \\
Tratamiento de la diabetes mellitus & $76(10,50 \%)$ & $126(17,00 \%)$ & \\
Dieta & $462(62,30 \%)$ & $382(51,51 \%)$ & $0,130 * *$ \\
Antidiabéticos orales & $201(27,10 \%)$ & $233(31,44 \%)$ & 0,03 \\
$\quad$ Tratamiento con insulina & $7,38 \pm 1,25$ & $8,38 \pm 1,55$ & \\
HbA1c media & & \\
\hline
\end{tabular}

* = Grado de significación de las medias mediante la aplicación del test t-Student; ** = la significación estadística hace referencia a las diferencias entre los pacientes insulino-tratados versus los no-insulino-tratados entre ambos estudios. 
Tabla II. Datos sobre la agudeza visual y tratamiento láser de los pacientes

Presente estudio

Agudeza visual
0,1
$0,2-0,4$
$>0,4$

Tratamiento láser

Panfotocoagulación

Fotocoagulación láser focal

\author{
$36(4,90 \%)(17,56 \%) *$ \\ $103(13,90 \%)(50,24 \%) *$ \\ $602(81,24 \%)$
}

$61(8,23 \%)(29,75 \%) * *$

$39(5,26 \%)(19,02 \%) * *$
Estudio del año 1993

* Porcentaje de pacientes con ceguera legal o baja visión respecto a la muestra de pacientes con retinopatía diabética; ** Porcentaje de pacientes tratados mediante fotocoagulación láser respecto al total de pacientes con retinopatía diabética.

patía diabética (se han excluido otras causas como catarata, atrofia de nervio óptico, etc.) han sido 103 pacientes $(13,90 \%)$, siendo la agudeza visual superior a 0,4 en 602 pacientes $(81,24 \%)$. El edema macular fue responsable de ceguera en 19 casos $(52,77 \%)$ y de baja visión en 40 casos $(38,83 \%)$.

En el estudio de 1993 la agudeza visual de los pacientes de la muestra fue: inferior o igual a 0,1 (ceguera legal) en el mejor ojo en 83 pacientes $(11,20 \%)$, los pacientes con baja visión secundaria a la presencia de retinopatía diabética fueron 143 pacientes $(19,29 \%)$, siendo la agudeza visual superior a 0,4 en 515 pacientes $(69,50 \%)$.

Si se compara el porcentaje de pacientes ciegos o con baja visión respecto al total de pacientes con retinopatía diabética (tabla II), se puede observar que el número de pacientes con ceguera ha disminuido de un $28,42 \%$ en el año 1993 a un $17,56 \%$ en la actualidad; este fenómeno no pasa respecto a los pacientes con baja visión que representan un $50,24 \%$ en la actualidad frente al $48,97 \%$ del año 1993.

\section{Estudio de prevalencia de retinopatía diabética}

La retinopatía se observó en 205 pacientes (27,55\%), siendo en el anterior estudio de 1994 de 292 pacientes $(39,41 \%)$. Según el tipo de retinopatía diabética observada, en el presente estudio 169 pacientes $(16,10 \%)$ presentan retinopatía de fondo leve, 21 pacientes $(2,80 \%)$ la forma moderada, $35(4,70 \%)$ la forma severa y $30(4,00 \%)$ la forma proliferativa. Las diferencias con el anterior estudio se pueden apreciar en la tabla III, en ella se puede ver que existe un descenso en el número de pacientes que presentan retinopatía diabética de fondo leve o intra retiniana, a su vez hay un descenso en el número de pacientes con retinopatía prolifertiva, pero existe un aumento de los que presentan retinopatía severa (preproliferativa).

\section{Estudio Univariante de retinopatía diabética (tabla IV)}

La aplicación del test de Chi-cuadrado demostró que el sexo $(\mathrm{p}=0.613)$ y la edad $(\mathrm{p}=0,123)$ no se

Tabla III. Prevalencia de retinopatía diabética y edema macular y clasificación de sus formas

\begin{tabular}{lcc}
\hline & Presente estudio & Estudio del año 1993 \\
\hline $\begin{array}{l}\text { Prevalencia de retinopatía diabética } \\
\text { Tipo de retinopatía diabética }\end{array}$ & $205 / 741(27,55 \%)$ & $292 / 741(39,41 \%)$ \\
$\quad$ de fondo leve / moderada* & $140(18,90 \%)$ & $220(29,68 \%)$ \\
de fondo severa ** & $35(4,70 \%)$ & $15(2,02 \%)$ \\
proliferativa & $30(4,00 \%)$ & $57(7,69 \%)$ \\
Prevalencia de edema macular & $59(7,90 \%)$ & $53(7,15 \%)$ \\
Tipo de edema macular & $49(6,60 \%)$ & $45(6,07 \%)$ \\
$\quad$ focal & $10(1,30 \%)$ & $8(1,08 \%)$ \\
difuso & & \\
\hline
\end{tabular}

* = Las forma leve y moderada del presente estudio se equiparan a la forma intra retinal del primer estudio; ** = La forma severa del presente estudio se equipara a la forma pre proliferativa del primer estudio 
Tabla IV. Estudio estadístico de la prevalencia de retinopatía diabética y edema macular en el presente estudio

Retinopatía diabética

Edema macular diabético

\begin{tabular}{|c|c|c|c|c|c|c|c|c|}
\hline \multirow[t]{2}{*}{ Factor de riesgo } & \multicolumn{2}{|c|}{ Chi-cuadrado } & \multicolumn{2}{|c|}{ Regresión logística } & \multicolumn{2}{|c|}{ Chi-cuadrado } & \multicolumn{2}{|c|}{ Regresión logística } \\
\hline & $\begin{array}{l}\text { Significación } \\
\text { (p) }\end{array}$ & $\begin{array}{l}\text { Odds } \\
\text { ratio }\end{array}$ & $\begin{array}{l}\text { Significación } \\
\text { (p) }\end{array}$ & $\begin{array}{l}\text { Odds ratio } \\
\text { Exp (B) }\end{array}$ & $\begin{array}{l}\text { Significación } \\
\text { (p) }\end{array}$ & $\begin{array}{l}\text { Odds } \\
\text { ratio }\end{array}$ & $\begin{array}{l}\text { Significación } \\
\text { (p) }\end{array}$ & $\begin{array}{l}\text { Odds ratio } \\
\operatorname{Exp}(\mathrm{B})\end{array}$ \\
\hline Sexo & 0,163 & 1,193 & 0,538 & 0,856 & 0,080 & 0,659 & 0.118 & 0,489 \\
\hline Edad & 0,123 & 1,193 & 0,114 & 1,420 & 0,190 & 1,102 & 0,334 & 2,126 \\
\hline Hipertensión arterial & 0,023 & 1,637 & 0,570 & 1,172 & 0,187 & 1,378 & 0,786 & 0,911 \\
\hline $\begin{array}{l}\text { Tiempo de evolución } \\
\text { de la diabetes mellitus }\end{array}$ & $<0,001$ & 12,541 & $<0,001$ & 5,898 & $<0,001$ & 5,667 & 0,394 & 1,327 \\
\hline $\begin{array}{l}\text { Niveles elevados } \\
\text { de } \mathrm{HbA} 1 \mathrm{c}>8 \%\end{array}$ & $<0,001$ & 22,540 & $<0,001$ & 19,022 & $<0,001$ & 12,748 & $<0,001$ & 9,043 \\
\hline $\begin{array}{l}\text { Niveles elevados de } \\
\text { colesterol-LDL }\end{array}$ & $<0001$ & 5,854 & 0,454 & 1,237 & $<0,001$ & 4,883 & 0,507 & 1,244 \\
\hline Tratamiento con insulina & $<0,001$ & 14,122 & $<0,001$ & 6,440 & $<0,001$ & 4,865 & 0,031 & 2,068 \\
\hline
\end{tabular}

relacionaban con la presencia de retinopatía diabética, siendo significativas la asociación con la hipertensión arterial $(\mathrm{p}=0,023)$, el tratamiento con insulina $(\mathrm{p}<0,001)$, el tiempo de evolución superior a los 15 años $(\mathrm{p}<0,001)$, los niveles elevados de HbA1c $(p<0,001)$ y los niveles elevados de colesterol-LDL $(\mathrm{p}<0,001)$.

\section{Estudio Multivariante de retinopatía diabética (tabla IV)}

La aplicación de la regresión logística a las distintas variables estudiadas dio como resultado que el sexo, la edad, la presencia de hipertensión arterial y los niveles elevados de colesterol-LDL no se relacionan significativamente con la aparición de retinopatía diabética; siendo significativos: el tiempo de evolución de la diabetes mellitus superior a 15 años $(\mathrm{p}<0,001)$, el tratamiento mediante insulina $(\mathrm{p}<0,001)$ y los niveles elevados de HbA1c ( $\mathrm{p}<0,001)$.

\section{Estudio de prevalencia de edema macular diabético}

El edema macular se observó en 59 pacientes $(7,90 \%)$, siendo la forma focal la más frecuente observándose en 49 pacientes $(6,60 \%)$, mientras que la forma difusa afectó a 10 pacientes $(1,30 \%)$.

Tabla V. Comparación de los resultados de prevalencia de retinopatía diabética en los distintos estudios publicados en España, se indican sólo los valores referidos a prevalencia de retinopatía en pacientes con diabetes mellitus tipo II

\begin{tabular}{|c|c|c|c|}
\hline Autor, Año & Población & Muestra & Prevalencia $(\%)$ \\
\hline Fernández Vigo 1990 & Galicia & 1.179 & 43,30 \\
\hline Martín 1992 & Extremadura & & 30,11 \\
\hline Romero 1994 & Tarragona & 741 & 39,41 \\
\hline Flores 1994* & Sevilla & 496 & 51,4 \\
\hline Goldaracena 1995 & Pamplona & 500 & 23,5 \\
\hline Romero 2000 & Riudoms (población semi urbana) & 283 & 24,4 \\
\hline Teruel 2005 & Girona & 401 & 34,29 \\
\hline Santos 2005 & Extremadura & 3.114 & 34,50 \\
\hline Esteban Ortega MM 1999** & Granada & 469 & $73,3 \%$ \\
\hline López IM 2002*** & Valladolid & 3.544 & $\begin{array}{c}\text { 20,41\% en tipo II: } \\
48,6 \% \text { (insulino tratados) } \\
14.7 \% \text { (no insulino tratados }\end{array}$ \\
\hline Presente estudio & Tarragona & 741 & 27,55 \\
\hline
\end{tabular}

\footnotetext{
* Incluye pacientes tipo I y tipo II; ** Estudio realizado en el norte de Granada en pacientes con más de 10 años de evolución de la diabetes melli-

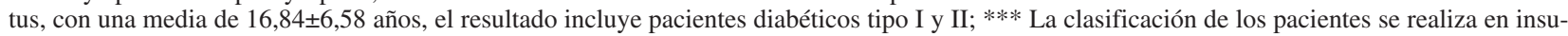
linodependientes y no insulinodependientes, no los clasifica en tipo I y tipo II, si bien deducimos la cifra de los datos de dicho estudio.
} 
Estudio Univariante de edema macular diabético (tabla IV)

La aplicación del test de Chi-cuadrado demostró que el sexo ( $\mathrm{p}=0,080)$, la edad $(\mathrm{p}=0,190)$, la presencia de hipertensión arterial $(\mathrm{p}=0,570)$ no se asociaban con en la presencia de edema macular, siendo significativos el tratamiento con insulina $(p<0,001)$, el tiempo de evolución superior a los 15 años $(\mathrm{p}<0,001)$, los niveles elevados de HbA1c $(\mathrm{p}<0,001)$ y los niveles elevados de colesterol-LDL $(\mathrm{p}<0,001)$.

\section{Estudio Multivariante de edema macular diabético (tabla IV)}

La aplicación de la regresión logística a las distintas variables estudiadas dio como resultado que el sexo, la edad, la presencia de hipertensión arterial, el tiempo de evolución de la diabetes mellitus, y los niveles elevados de colesterol-LDL no se asociaban significativamente con la aparición de retinopatía diabética, siendo significativos: el tratamiento mediante insulina $(\mathrm{p}=0,031)$ y los niveles elevados de HbA1c ( $\mathrm{p}<0,001)$.

Los pacientes de la muestra tratados previamente mediante fotocoagulación láser fueron (tabla II):

1. Pacientes tratados mediante panfotocoagulación láser: en el presente estudio en 61 casos $(8,23 \%)$ se observó la presencia de tratamiento mediante panfotocoagulación láser, la indicación de fotocoagulación láser fue la presencia de retinopatía diabética severa en 33 casos y la presencia de retinopatía proliferativa en 27 pacientes. En el anterior estudio (1993), en el momento de la realización del mismo solo en 28 pacientes $(3,77 \%)$ se había realizado tratamiento mediante panfotocoagulación láser, en seis casos por retinopatía diabética severa (preproliferativa, según la clasificación entonces existente) y en 16 casos por presentar retinopatía diabética proliferativa.

2. Fotocoagulación láser focal: en el presente estudio en 39 pacientes $(5,26 \%)$ se observó la presencia de tratamiento láser focal previo al mismo, en el anterior estudio se observó la presencia de fotocoagulación láser focal en 18 casos $(2,43 \%)$ previamente al estudio.

El estudio estadístico de los pacientes tratados mediante panfotocoagulación láser previamente al estudio demuestra que: el sexo no es un factor significativo en el mismo $(p=0,529 \mathrm{OR}=0,601-1,725)$ como tampoco lo son la edad $(\mathrm{p}=0,08 \mathrm{OR}=0,778$ $1,091)$ ni la presencia de hipertensión arterial $(\mathrm{p}=$ $0,319$ OR $=0,673-2,157)$, si son significativos el tiempo de evolución de la diabetes mellitus $(\mathrm{p}<$ $0,001 \mathrm{OR}=4,978-15,884)$, los niveles elevados de HbA1c $(\mathrm{p}<0,001 \mathrm{OR}=8,110-40,515)$ y el tratamiento mediante insulina ( $\mathrm{p}<0,001$ OR 7,29-27,116).

\section{DISCUSIÓN}

En España se han realizado múltiples estudios epidemiológicos con el objetivo de determinar la prevalencia de retinopatía diabética, así como los factores de riesgo con ella asociados (tabla V). En un primer momento los estudios adolecían de haberse realizado en hospitales y no eran representativos de la realidad poblacional. Así Pastor en Navarra en 1980 (1) obtenían una cifra de un 43,80\% de prevalencia de retinopatía diabética y Barahona en Salamanca (2) en 1985 una cifra de un 53,30\%. Uno de los problemas más importantes al intentar comparar estudios lo encontramos en la diferente metodología empleada en ellos, así aún hay estudios recientes que recurren a la oftalmoscopía directa para el diagnóstico de la retinopatía diabética.

Posteriormente fueron apareciendo nuevos estudios basados en poblaciones, que fueron rebajando las cifras de prevalencia así Fernández Vigo $(3,4)$ obtenía una cifra de un $43,30 \%$ en Galicia y el mismo autor obtenía una cifra de un 30,11\% en Extremadura. Nosotros realizamos en el año 1993 un estudio poblacional de prevalencia de retinopatía diabética en el área de influencia de nuestro Hospital $(6,8)$, obteniendo una cifra de prevalencia de un $39,42 \%$ de pacientes con diabetes mellitus tipo II y con retinopatía diabética. En el año 1999 realizamos también un estudio de prevalencia en una de la Áreas Básicas dependientes de nuestro Hospital (9), en la que se incluyeron el $92,78 \%$ de los diabéticos tipo II censados en dicho Centro de Atención Primaria, siendo el resultado de prevalencia de retinopatía diabética de un $24,40 \%$, esta cifra era muy inferior a la del primer estudio, y nos indujo a realizar un nuevo estudio con características similares al primero de 1993, para ello esperamos que se desplegaran todas la Áreas Básicas pendientes, y que estas aplicaran la nuevas recomendaciones diagnóstica de diabetes mellitus realizadas desde la OMS.

En lo que respecta al presente estudio se debe resaltar que reúne una serie de características 
importantes: Se ha realizado sobre la misma población de referencia que el primero en el año 1993, la metodología empleada para seleccionar la muestra ha sido la misma, e igualmente el diagnóstico de retinopatía diabética ha sido realizado empleando la misma técnica. Finalmente el equipo investigador ha sido el mismo en lo que aa máximos responsables del mismo se refiere.

De los datos obtenidos llama la atención que la prevalencia de retinopatía diabética ha descendido del valor en el año 1993 de un 39,41\% al 27,55\% en la actualidad, este hecho creemos que se puede deber a las siguientes causas: en primer lugar en el año 1993 no estaban abiertas todas la Áreas Básicas existentes en la actualidad, con lo que el diagnóstico y control de los pacientes diabéticos, por parte de los médicos de atención primaria, era menor que en la actualidad; se puede suponer que en la actualidad la parte de población con lo que se denominaba como diabetes no conocida, ha ido aflorando y siendo diagnosticada; en segundo lugar los criterios de diagnóstico de diabetes mellitus han sido cambiados en el año 1998, lo que ha llevado a la aparición de una mayor población diabética, que en su mayor parte se controla con dieta o antidiabéticos orales, finalmente creemos que el mayor control ejercido sobre los pacientes con diabetes mellitus, es posible que haya disminuido la aparición de complicaciones crónicas como la retinopatía diabética.

La cifra obtenida en el presente estudio $(27,55 \%)$ en los pacientes con diabetes mellitus tipo II, es inferior a la obtenida en recientes estudios realizados en nuestro país y con base poblacional, como son el de Santos en Extremadura (13) que da una cifra de $34,50 \%$ y el de Teruel-Macias en Girona (14) con una cifra de un 34,29\%, solamente: Goldaracena obtiene una cifra de prevalencia inferior a la nuestra siendo de $23,5 \%$ en su estudio en Pamplona del año 1995 (11) y López en Valladolid en el año 2002 (12) obtiene una cifra de un 20,41\% en pacientes diabéticos tipo II, si bien este último el tiempo de evolución medio de la diabetes mellitus es de solo 5 años; de todas maneras en su conjunto se puede observar que las cifras de prevalencia de retinopatía diabética han ido disminuyendo progresivamente en todos los estudios.

Respecto a las diferencias observadas en los resultados con el primer estudio del año 1993 podemos observar que, en lo que a pérdida de agudeza visual se refiere fue inferior o igual a 0,1 (ceguera legal secundaria a la retinopatía diabética) en el mejor ojo en 36 pacientes $(4,90 \%)$, y han sido 103 pacientes $(13,90 \%)$ los afectados de baja visión secundaria a la presencia de retinopatía diabética. Estas cifras serían mejores que las obtenidas en el primer estudio donde la ceguera legal (AV $\leq 0,1)$ estaba presente en un $11,27 \%$ de casos y la presencia de baja visión (AV 0,2-0,4) afectaba a un $19,40 \%$ de casos, pero hemos de indicar que si bien podemos en parte relacionar la mejoría de la agudeza visual con un mejor control de los pacientes diabéticos, estas cifras estarían también afectadas por un efecto producido por un mayor número de pacientes diagnosticados de diabetes mellitus y por tanto una disminución relativa de los afectados de pérdida de agudeza visual respecto al total de pacientes diabéticos.

Tal vez es más interesante el observar el tipo de retinopatía diabética que presentan los pacientes, así podemos ver que la presencia de pacientes con retinopatía diabética proliferativa ha disminuido de un 57 pacientes $(7,69 \%)$ a $30(4,00 \%)$, habiendo aumentado la presencia de pacientes con retinopatía de fondo severa (preproliferativa según la definición del primer estudio) pasando de 15 pacientes $(2,02 \%)$ a 35 pacientes $(4,70 \%)$; este hecho podría hacernos pensar que se están realizando diagnósticos en fases más precoces de la retinopatía diabética y por tanto mejorando las posibilidades de tratamiento de la misma. Se puede destacar así mismo que el número de pacientes con retinopatía de fondo leve-moderada (intra retinal) es menor en el presente estudio, esto podría llevar a pensar que un mejor control de la diabetes disminuye el número de pacientes con retinopatía, pero este dato debe ser tomado con cautela, ya que seguramente ha aumentado el número de pacientes diagnosticados de diabetes mellitus.

Igualmente interesante es observar el número de tratamientos láser realizados previamente en los pacientes de las dos muestras, así el número de pacientes tratados mediante panfotocoagulación láser es de 61 casos $(8,23 \%)$, mientras que en el anterior estudio (1993) fue solo de 28 pacientes $(3,77 \%)$, igualmente el número de pacientes tratados mediante fotocoagulación láser focal también era inferior en el estudio del año 1993 siendo 18 casos $(2,43 \%)$ frente a los 39 pacientes $(5,26 \%)$ en el actual. Este hecho corroboraría la idea de que existe un mayor control sobre los pacientes diabéticos.

Respecto a los factores de riesgo que influyen en el desarrollo de la retinopatía diabética hemos 
observado los ya descritos profusamente en la literatura, así son significativos: el tiempo de evolución de la diabetes mellitus, el mal control metabólico de la misma (evaluado a través de los niveles de HbA1c) y el tratamiento mediante insulina.

Respecto a la prevalencia de edema macular diabético actual, es similar a la de nuestro primer estudio (7,30\% en el actual y 7,15\% en el del año 1993), estos valores son algo superiores a algunos estudios publicados en nuestro país, así López (12) encuentró una prevalencia de $5,7 \%$ en Valladolid y Santos (13) una prevalencia de 5,6\% en Extremadura y similar a la encontrada por Flores en Sevilla con un 7,5\% (7); si bien hemos de destacar que existen pocos datos epidemiológicos publicados sobre edema macular diabético. Del estudio estadístico de los factores de riesgo solamente los niveles elevados de HbA1c y el tratamiento con insulina, son significativos, hemos de destacar que ambos factores se pueden relacionar con un mal control de la diabetes mellitus.

Es pues muy importante el estricto control de la glicemia en los pacientes diabéticos como ponen de manifiesto diversos estudios (17,23-25). Entre ellos el DCCT (17) que demostró que un estricto control de la glucemia, durante un periodo prolongado, reduce la incidencia de edema macular, con un riesgo relativo de un 0,78 , siendo el porcentaje de cambio de $-22,1 \%$. No obstante el mismo estudio puso también en evidencia, que en el grupo de pacientes sometidos a un estricto control de la glucemia (bien mediante el uso de bomba de insulina o bien múltiples inyecciones de insulina), y sobre todo en el subgrupo de pacientes con niveles elevados de glucemia, aparecía edema macular durante el primer año en un $2,78 \%$ frente a un $1,72 \%$ en el grupo de pacientes sometidos a control convencional de la diabetes mellitus (26). Este empeoramiento ya no existía en el siguiente periodo de tiempo así a partir del primer año y hasta el tercer año de seguimiento, la presencia de edema macular se producía en un $3,58 \%$ de casos en el grupo de pacientes con tratamiento convencional frente a un $2,61 \%$ en el grupo con tratamiento intensivo. Se puede concluir pues que un control estricto instaurado bruscamente en los pacientes diabéticos puede desencadenar la aparición de edema macular, siendo importante evitarlo.

En conclusión atendiendo a los resultados del presente estudio, se puede observar un descenso en la prevalencia de la retinopatía diabética, pero creemos que este hecho se puede deber más al hecho de un aumento de la población de pacientes diabéticos conocidos, que solamente al mejor control ejercido sobre los mismos. De todas maneras parece que podemos observar una mejoría en las características de nuestros pacientes, ya que existe un descenso en el número de pacientes con ceguera y un mayor número de pacientes tratados correctamente. A pesar de todo la retinopatía diabética sigue siendo una causa importante de disminución de la agudeza visual como lo atestiguan el $4,9 \%$ de pacientes ciegos y el 13,9\% con baja visión, relacionadas ambas con la retinopatía diabética.

\section{BIBLIOGRAFÍA}

1. Pastor Jimeno JC, Eder Laibaiburu F, Terkawi Y. El diabético de alto riesgo. Estudio epidemiológico de la microangiopatia retiniana en 500 diabéticos. Arch Soc Esp Oftalmol 1980; 40: 103-114.

2. Barahona Hortelano JM, Alió y Sanz. JL. Epidemiología y prevención de la ceguera por diabetes mellitus en nuestro medio. Arch Soc Esp Oftalmol 1985; 48: 27-46.

3. Sández Macho J, Fernández-Vigo J. Estudio epidemiológico de campo sobre prevalencia de retinopatía diabética simple y ceguera en una población diabética. Arch Soc Esp Oftalmol 1991; 60: 419-424.

4. Fernández-Vigo J. La diabetes ocular en cifras. Diabetes ocular. Barcelona: Edika Med; 1992.

5. Martín C, Fernández-Vigo J, Fernández J, Díaz AJ, Montero I, Molina M. Prevalencia de retinopatía diabética: estudio comparativo de dos poblaciones no seleccionadas en Galicia y Extremadura. Arch Soc Esp Oftalmol 1992; 62: 389-394.

6. Romero Aroca P. Prevalencia de retinopatía diabética y ceguera visual en la población del Baix Camp (Tarragona). Tarragona: Universidad Rovira i Virgili. 1994. 239 pp. Tesis Doctoralis.

7. Flores Vicedo CM, Castellanos Mateos, Piñero Bustamante A. Retinopatía diabética. Situación de nuestra área hospitalaria. Arch Soc Esp Oftalmo 1996; 71: 65-72.

8. Romero Aroca P, Del Castillo Dejarin D. Estudio de prevalencia de retinopatía diabética en la población del Baix Camp (Tarragona). Arch Soc Esp Oftalmol 1996; 71: 261 268.

9. Romero Aroca P, Calviño Domínguez O, del Castillo Dejardin D. Estudio epidemiológico de retinopatía diabética, en un área básica de salud. Arch Soc Esp Oftalmol 2000; 75: 147-152.

10. Esteban Ortega MM, Rodríguez Hurtado F, Jiménez Monleón JJ, Bueno Cavanillas A. Prevalencia de la retinopatía en diabéticos de más de diez años de evolución en la zona Norte de Granada. Arch Soc Esp Oftalmol 1999; 74: 137-144.

11. Goldaracena MB, Escudero JM, Arrondo A, Vilarubia A, Aramendia $B$, Iturralde $R$. Prevalencia de retinopatía diabética en una población diabética registrada en atención primaria. Arch Soc Esp Oftalmol 1998; 73: 263-268. 
12. Lopez IM, Diez, A, Velilla S, Rueda A, Alvarez A, Pastor $C J$. Prevalence of diabetic retinopathy and eye care in a rural area of Spain. Ophthalmic Epidemiol 2002; 9: $205-$ 214.

13. Santos Bueso E, Fernández-Vigo J, Fernández Pérez C, Macarro Merino A, Fernández Perianes J. Prevalencia de retinopatía diabética en la Comunidad Autónoma de Extremadura. 1997-2001 (Proyecto Extremadura para prevención de la ceguera). Arch Soc Esp Oftalmol 2005; 80: 187-194.

14. Teruel Macias C, Fernández-Real JM, Ricart W, Valent Ferrer $R$, Vallés Prats $M$. Prevalencia de la retinopatía diabética en la población de diabéticos diagnosticados en las comarcas de Girona. Estudio de los factores asociados. Arch Soc Esp Oftalmol 2005; 80: 85-92.

15. World Health Organization. Diabetes Mellitus. Report of a WHO Study Group Geneva. World Health Org. 1985 (Tech Rep Ser no 727).

16. The Expert Committee on the Diagnosis and Classification of Diabetes Mellitus. Report of the Expert Committee on the Diagnosis and Classification of Diabetes Mellitus. Diabetes Care 2000; 23: S4-S19.

17. Diabetes Control and Complications Trial Research Group. Progression of retinopathy with intensive versus conventional treatment in the Diabetes Control and Complications Trial. Ophthalmology 1995; 102: 647-661.

18. Kohner EM, Aldington SJ, Stratton IM, Menley SE, Holman RR, Turner RC. United Kingdom Prospective Diabetes Study, 30: diabetic retinopathy at diagnosis of noninsulin-dependent diabetes mellitus and associated risk factors. Arch Ophthalmol 1998; 116: 297-303.
19. Castell C, Tresserras R, Serra J, Goday A, Lloveras G, Salleras L. Prevalence of diabetes in Catalonia (Spain): an oral glucose tolerance test-based population study. Diabetes Res Clin Pract 1999; 43: 33-40.

20. Aldington SJ, Kohner EM, Meuer S, Klein R, Sjolie AK. Methodology for retinal photography and assessment of diabetic retinopathy: The EURODIAB IDDM complications study. Diabetologia 1995; 38: 437-444.

21. Early Treatment Diabetic Retinopathy Study Research Group. Grading diabetic retinopathy from stereoscopic color fundus photographs--an extension of the modified Airlie House classification. ETDRS report number 10. Ophthalmology 1991; 98: 786-806.

22. Wilkinson CP, Ferris FL 3rd, Klein RE, Lee PP, Agardh $C D$, Davis $M$, et al. Proposed international clinical diabetic retinopathy and diabetic macular edema disease severity scales. Ophthalmology 2003; 110:1677-1682.

23. Klein R, Klein BE, Moss SE, Cruickshanks KJ. The Wisconsin Epidemiology Study of Diabetic Retinopathy. XV. The long-term incidence of macular edema. Ophthalmology 1995; 102: 7-16.

24. Do DV, Shah SM, Sung JU, Haller JA, Nguyen QD. Persistent diabetic macular edema is associated with elevated hemoglobin A1c. Am J Ophthalmol 2005; 139: 620-623.

25. Williams R, Airey M, Baxter H, Forrester J, KennedyMartin T, Girach A. Epidemiology of diabetic retinopathy and macular oedema: a systematic review. Eye 2004; 18: 963-983.

26. Early worsening of diabetic retinopathy in the Diabetes Control and Complications Trial. Arch Ophthalmol 1998; 116: 874-886. 